\title{
Management of liver complications in haemoglobinopathies
}

\author{
Pierre Brissot, Olivier Loreal \\ Service des Maladies du Foie, Hôpital Pontchaillou, Rennes University Hospital, Rennes, France
}

\begin{abstract}
Liver complications in haemoglobinopathies (thalassaemia and sickle cell disease) are due to several factors, dominated (beside chronic viral infections, not considered here) by chronic iron overload, biliary obstruction and venous thrombosis. Whereas the latter two factors can cause acute hepatic syndromes, all three mechanisms - when becoming chronic- can produce fibrosis and cirrhosis and even, in thalassaemia, hepatocellular carcinoma. These chronic hepatic complications are an indirect consequence of the significant improvement in life expectancy due to the overall amelioration of disease management. The diagnostic approach has benefited from non invasive (biochemical and imaging) approaches which have considerably reduced the indication of liver biopsy. The therapeutic management involves relatively efficient curative medical, endoscopic or surgical methods, but should rest primarily on preventive measures focused on the haematological causative factors but also on hepatic co-morbidities. This chapter will focus on hepatic complications in thalassaemia and sickle cell disease (SCD), without considering the complications related to virus $\mathrm{B}$ or $\mathrm{C}$ infections which will be described in another chapter.
\end{abstract}

\section{Background}

\section{Harmful factors for the liver}

\section{Factors related to haematological disease}

Iron overload

In thalassaemia: ${ }^{1}$ Repeated transfusions represent the major cause of iron overload in thalassemia major(TM). Each blood unit corresponds to $200-250 \mathrm{mg}$ of iron. Considering that total body iron stores approximate $4 \mathrm{~g}$ and that normal daily iron losses are of the order of 1-

\footnotetext{
E-mail: pierre.brissot@univ-rennesl.fr iron overload; hepatocellular carcinoma.

CC Copyright P. Brissot and O. Loreal, 2014

Licensee PAGEPress, Italy

Thalassemia Reports 2014; $4: 4869$

doi:10.4081/thal.2014.4869
}

Correspondence: Pierre Brissot, Service des Maladies du Foie, Hôpital Pontchaillou, Rennes University Hospital, Rennes, France

Key words: thalassaemia; sickle cell diseas; hemoglobinopathies; chronic

This article is distributed under the terms of the Creative Commons Attribution Noncommercial License (by-nc 3.0) which permits any noncommercial use, distribution, and reproduction in any medium, provided the original author(s) and source are credited.
$2 \mathrm{mg}$ (with a very limited capacity for the body to regulate these losses), one can understand that, when a given individual needs, for instance, one unit of blood every 2 weeks, body iron overload develops rapidly. Since red blood cells are degraded in the reticulo-endothelial system (macrophages, essentially within the spleen), iron overload will primarily affect the spleen and, at a lesser degree, hepatic macrophages (called Kupffer cells). Thereafter, intramacrophagic iron will be released progressively into the blood stream, in order to reach the bone marrow and lead to the production of new red blood cells. During this releasing process, plasma transferrin - the normal iron transport protein in the blood, whose normal saturation by iron is less than $45 \%$ - increases rapidly, reaching often $100 \%$. This will lead to the appearance of plasma non-transferrin bound iron (NTBI), ${ }^{2}$ an iron species which has the property to be very rapidly taken up by parenchymal cells (the hepatocytes for the liver), therefore accounting primarily for hepatic iron overload, the liver being, for circulating iron, both the first line target and the main storage organ.

Dyserythropoiesis is another important mechanism explaining iron excess. It acts through a decreased production of the iron regulatory hormone hepcidin by the liver. Hepcidin deficiency leads to an abnormal maintenance of the cellular iron exporter (ferroportin) activity. ${ }^{3}$ In turn, this favors an increased iron entry into the plasma at two major sites: on one hand, the duodenum corresponding to an increased intestinal absorption of iron ; on the other hand, and quantitatively 10 to 20 times more important, at the splenic level. This leads to increased transferrin saturation, with the appearance of plasma nontransferrin bound iron (NTBI). The intimate mechanism whereby dyserythropoiesis favors iron excess through hepcidin deficiency has known recently a major breakthrough with the discovery of the hormone erythroferrone which corresponds very likely to the long-sought "erythropoietic factor". 4 The role of dyserythropoiesis as a cause for iron excess can be considered relatively accessory as compared to transfusional iron intake in case of TM (where it however may explain that these patients can develop significant iron overload before any transfusion). In contrast, dyserythropoiesis is the primary iron overloading factor in non-transfusion dependent thalassemia, ${ }^{5,6}$ where iron pathophysiology is in fact very close to that of hepcidin deficient hereditary hemochromatosis. ${ }^{7}$

In sickle cell disease: A study by Vichinsky et al. ${ }^{8}$ concluded that the average rate of iron loading due to transfusions was approximately half in SCD. However, because of the repeated transfusions prescribed over a lifetime both to treat SCD crises (strokes, acute chest syndrome, splenic sequestration) and to prevent those crises, significant iron overload can develop in a number of patients. ${ }^{9}$ A recent prospective study on 35 SCD patients indicated that the median hepatic iron content was similar in SCD and TM. ${ }^{10}$ Plasma NTBI has been documented in SCD. ${ }^{11}$

Ineffective erythropoiesis is much less involved in SCD as compared to TM. Indeed, whereas erythrocyte degradation takes place predominantly in the bone marrow in $\mathrm{TH}$, leading to ineffective erythropoiesis, it occurs primarily within the vascular system in SCD. ${ }^{9}$

Another difference with TM is the inflammatory state often present in SCD. Being responsible for an increased hepcidin synthesis, inflam- 
mation may limit the duodenal absorption of iron and iron release from the spleen (in non splenectomized subjects), and therefore favors macrophagic iron overloading.

\section{Biliary obstruction}

In thalassaemia: A multicenter study in $858 \mathrm{TM}$ patients concluded to a remarkable prevalence (30\%) of cholelithiasis. ${ }^{12,13}$ Increased bilirubin production, related to chronic hemolysis, is the primary cause, but the presence of a Gilbert syndrome genotype (resulting in the decreased production of conjugated bilirubin) favors ${ }^{14}$ both the frequency of gallstones and the age at which it develops. Although cholelithiasis, when remaining located within the gladder, has no damaging effect on the liver itself, it is no more the case when gallstones migrate into the biliary duct (choledocolithiasis), being responsible for cholestasis and infection.

In sickle cell disease: Cholelithiasis has been reported in nearly $60 \%$ of SCD patients, with $17 \%$ having concomitant choledocolithiasis. ${ }^{14,15}$

\section{Vascular obstruction}

In thalassaemia: The prevalence of overall thromboembolic complications was $1.6 \%$ in a population of 860 patients, and was four-times as high in thalassemia intermedia as compared to TM. ${ }^{13,16}$ Venous thromboembolism was more frequent than arterial thromboembolism for both groups of patients. The main underlying mechanism is an hypercoagulable state due to several factors (abnormal red blood cell surface, platelet activation, and endothelial cell activation). ${ }^{17}$ Splenectomy is an expected risk factor, due to thrombocytosis and increased abnormal erythrocytes in the circulation. It has been reported that, in TM, splenic weight and laparoscopic surgery were risk factors for portal vein thrombosis. ${ }^{18}$ Cases of hepatic vein thrombosis (Budd-Chiari syndrome) have been described in TM. ${ }^{19}$

In sickle cell disease: Naik RP et al. recently reported in 1,523 SCD patients, aged $\geq 15$ years and cumulating 8,862 years of follow-up, an incidence rate for first venous thromboembolism of 5.2 events/1000 person-years, and a cumulative incidence of $11.3 \%$ by age $40 .^{20}$

A specific vascular-related hepatic complication, named sickle cell intrahepatic cholestasis (SCIC), has been described in SCD. It may be mainly due to trapping of sickled erythrocytes within the hepatic sinusoids with subsequent localized hypoxia, causing hepatocyte ballooning which in turn would lead to a back pressure effect, causing intracanalicular cholestasis. ${ }^{21,22}$

\section{Extra-haematological factors}

It is expected that the various factors which can, by themselves, damage the liver, may aggravate liver dysfunction when associated with thalassaemia or sickle cell disease. Those conditions are especially (besides HBV or HCV chronic infections) non alcoholic fatty liver disease (NAFLD), ${ }^{23}$ chronic alcoholism, or xenobiotics.

\section{Hepatic patho-physiological consequences}

\section{Iron overload}

As long as iron excess is limited to macrophages, hepatic damage is relatively limited because iron seems less toxic when deposited within the reticulo-endothelial cells (ref). Hepatocytic iron overload, in contrast, is potentially highly damaging. Indeed, as soon as the protective effect of the iron storage protein ferritin is exceeded, hepatocyte damage occurs leading to cellular necrosis followed by the progressive scarring process of fibrosis, whose ultimate stage is cirrhosis. The way iron toxicity develops is largely dependent on NTBI. Indeed, part of this iron species exists under the form of labile plasma iron (LPI $)^{24-26}$ which has a high propensity to produce reactive oxygen species (ROS). ROS are known to damage membrane lipids, affecting not only plasma membranes of the hepatocytes but also the membranes of intracellular organites, including the nuclei. In SCD, oxidative stress has also been reported. ${ }^{27}$ It may be related not only to tissue iron excess but also to intravascular hemolysis through heme-induced endothelial toxicity. ${ }^{9}$ On the long-term, the risk is that of fibrosis and cirrhosis. ${ }^{9,13}$

\section{Biliary obstruction}

Choledoco-lithiasis can lead to biliary stasis (cholestasis). Acute cholestasis may transiently damage the hepatocytes, creating what could be named a «biliary hepatitis». The other risk is the development of infection (cholangitis with the possibility of septicemia). Chronic cholestasis can produce fibrosis and end up with cirrhosis (biliary cirrhosis).

\section{Vascular obstruction}

Portal venous thrombosis: It can be responsible for portal hypertension, with its risk of digestive haemorrhage by rupture of oesophagealgastric varices.

Hepatic vein thrombosis: It can cause acute or chonic congestive liver, with the risks of liver failure and chronic fibrosis.

In summary, most aggressions towards the liver lead to hepatocyte necrosis, source of cellular regeneration but also of fibrosis. When the hepatic insult lasts for a long time, which is more and more frequent given the major improvement in life expectancy in thalassaemia, cirrhosis can develop with its own risk for hepatocellular carcinoma, especially when associated with chronic viral C or B infection. ${ }^{28,29}$

\section{Diagnostic management of liver complications}

The diagnosis of hepatic complications is a multistep process.

\section{Clinical examination}

It should always remain the first step, which precedes and orientates biochemical, and morphological investigations.

Its goal is to collect data reflecting hepatic morphology and dysfunction .

\section{Morphological data}

The examination should search for hepatomegaly and evaluates its consistency, regularity, and sensitivity. Thus, clinical assessment alone may, for instance, strongly suggest a cirrhotic liver.

\section{Functional data}

Whatever the liver size, it is essential to search for possible signs of hepatic dysfunction. Three main sets of data should be looked for: i) signs of hepato-cellular failure (hepatocytic defective synthesis): spider angiomas, palmar erythema, bruises, finger clubbing; ii) signs of cholestasis: pruritis, jaundice; iii) signs of portal hypertension: abdominal collateral venous circulation; splenomegaly (may be difficult to interpret in the haematological context); iv) signs resulting from both hepatocellular failure and portal hypertension: ascites, hepatic encephalopathy.

\section{Biochemical assessment}

Its involves the «classical» liver function tests, but also emerging «morphological» bio-markers.

\section{Liver function tests}

Three main biological syndromes need to be evaluated and confronted to the clinical data: i) cytolysis: expressed by an increase of serum transaminase activities (ALT -alanine aminotransferase, AST -aspartate aminotransferase, keeping in mind that hemolysis can increase AST; ${ }^{30}$ ii) cholestasis: combined increase of serum alkaline phosphatase and GGT ( $\gamma$ glutamyltransferase); elevated serum conjugated bilirubin; iii) hepato-cellular failure: increased prothrombin time (PT) or international normalized ratio (INR), low serum albumin. 


\section{Morphological biomarkers}

They consist of serum markers aiming to reflect hepatic fibrosis. Hyaluronic acid concentration, as a single test, may be helpful. ${ }^{31,32}$ However, the most promising tests are composite ones, such as the Fibrotest ${ }^{\circledR}$. It should be kept in mind that the latter test is mostly valid for distinguishing between, on one hand, cirrhosis (or major fibrosis), and on the other hand, absence (or minimal) fibrosis, and that it has been essentially validated in the context of chronic hepatitis $\mathrm{C}$. The scores, including platelet counts such as the Apri test and Fib-4 test may not be appropriate, given the haematological context most likely to impact the platelets. ${ }^{33}$

\section{Imaging techniques}

Ultrasound (echo-Doppler) examination: It is the first line technique, providing not only morphological details on the liver, biliary tract, portal and hepatic vein patency, but also functional informations when detecting signs of portal hypertension.

Scanner and MRI (magnetic resonance imaging) are indicated according to the ultrasound data: For instance, an angio-scanner will be performed to further explore a nodule, and MRI is especially justified to visualize the biliary tract (independently of the MRI interest for quantifying hepatic and splenic iron load).

Transient elastography: This recent technique evaluates, in a non invasive way, the degree of hepatic fibrosis. Its interest has been documented in thalassaemia. ${ }^{33-36}$ One must be aware, however, that transient elastography explores the elasticity of the liver and not directly the fibrosis state, and therefore that there exist false-positive situations such as acute hepatitis or liver venous congestion (as reported during sickle cell vaso-occlusive crisis $)^{37}$.

Liver biopsy: Although still considered as the reference method for assessing iron overload, and for staging hepatic fibrosis and chronic hepatitis, and reported as a globally safe procedure, ${ }^{38,39}$ it remains an invasive technique, especially in these haematological diseases with coagulation disorders. Resorting to transjugular venous biopsy (instead of the transparietal approach) could minimize the haemorrhagic hazard but may not be appropriate in case of hypercoagulable state. Liver biopsy is therefore increasingly replaced by the combination of biochemical tests and imaging techniques (serum ferritin + MRI for iron status, fibrosis biomarkers + transient elastography for fibrosis).

\section{Therapeutic management of liver complications}

\section{Curative management}

\section{Iron overload}

Deferasirox has been the most widely studied oral chelator, especially for evaluating the evolution of hepatic fibrosis. ${ }^{38}$ Given in 219 thalassemia patients for 3 years or more, deferasirox reversed or stabilized liver fibrosis in $83 \%$ of patients. Unexpectedly, the positive effect on fibrosis was not strictly correlated with the degree of iron depletion, raising the issue of a specific anti-fibrogenic effect of this compound (independent of its iron chelating property). Moreover, this therapeutic effect was independent of previous exposure to HCV.

\section{Biliary obstruction}

Surgical (especially laparoscopic) or endoscopic procedures have no specificity to treat choledocolithiasis, ${ }^{15}$ with special emphasis on antibiotic treatment, given the susceptibility of the patients (especially after splenectomy) ${ }^{40}$ for infections.

\section{Venous thrombosis}

It is an indication for anticoagulants, followed up with special attention in this haematological context.

\section{Severe liver failure will benefit from general intensive care} management

Liver transplantation ${ }^{22,41}$ has been rarely performed but may become a growing therapeutic procedure, especially for management of hepatocellular carcinoma, ${ }^{42}$ in highly selected cases.

\section{Preventive management}

It constitutes a major part of the overall therapeutic management, focused on preventing iron overload by early chelation, venous thrombosis by discussing anti-aggregative agents and/or anticoagulants before surgery and during pregnancy, ${ }^{43}$ and environmental co-morbidities by counteracting the metabolic syndrome and alcoholism. Moreover, in cirrhotic patients, it is essential to check systematically every 6 months serum $\alpha$-foetoprotein and hepatic ultrasound in order to detect an emerging hepatocellular carcinoma.

\section{Conclusions}

In conclusion, hepatic complications are a serious concern in haemoglobinopathies, dominated, beside chronic viral infections, by the consequences of iron overload, biliary obstruction and portal vein thrombosis. The development of cirrhosis, exposing to the risk of hepatocellular carcinoma, is an indirect consequence of the major improvement in life expectancy in these diseases, and represents a powerful incentive to adopt preventive measures against the various factors which can be responsible for acute and/or chronic hepatic damage.

\section{References}

1. Brissot P. The liver in thalassaemia. Guidelines for the Clinical Management of Thalassaemia. 2nd update ed.; 2014.

2. Brissot P, Ropert M, Le Lan C, Loreal 0. Non-transferrin bound iron: a key role in iron overload and iron toxicity. Biochimica et biophysica acta 2012;1820:403-10.

3. Ganz T, Nemeth E. Hepcidin and iron homeostasis. Biochimica et biophysica acta 2012;1823:1434-43.

4. Kautz L, Jung G, Nemeth E, Ganz T. Erythroferrone contributes to recovery from anemia of inflammation. Blood 2014;124:2569-74.

5. Taher AT, Viprakasit V, Musallam KM, Cappellini MD. Treating iron overload in patients with non-transfusion-dependent thalassemia. American journal of hematology 2013;88:409-15.

6. Musallam KM, Cappellini MD, Wood JC, Taher AT. Iron overload in non-transfusion-dependent thalassemia: a clinical perspective. Blood rev 2012;26 Suppl 1:S16-9.

7. Brissot P, Bardou-Jacquet E, Jouanolle AM, Loreal O. Iron disorders of genetic origin: a changing world. Trends in molecular medicine 2011;17:707-13.

8. Vichinsky E, Onyekwere 0 , Porter J, et al. A randomised comparison of deferasirox versus deferoxamine for the treatment of transfusional iron overload in sickle cell disease. $\mathrm{Br} \mathrm{J}$ Haematol 2007;136: 501-8.

9. Porter J, Garbowski M. Consequences and management of iron overload in sickle cell disease. Hematology Am Soc Hematol Educ Program 2013;2013: 447-56.

10. Hankins JS, Smeltzer MP, McCarville MB, et al. Patterns of liver iron accumulation in patients with sickle cell disease and thalassemia with iron overload. Eur J Haematol 2010;85:51-7.

11. Inati A, Musallam KM, Cappellini MD, et al. Nontransferrin-bound 
iron in transfused patients with sickle cell disease. Int $\mathrm{J}$ Lab Hematol 2011;33:133-7.

12. Origa R, Galanello R, Perseu L, et al. Cholelithiasis in thalassemia major. Eur J Haematol 2009;82:22-5.

13. Borgna-Pignatti C, Gamberini MR. Complications of thalassemia major and their treatment. Expert Rev Hematol 2011;4:353-66.

14. Bond LR, Hatty SR, Horn ME, et al. Gall stones in sickle cell disease in the United Kingdom. Br Med J (Clin Res Ed) 1987;295:234-6.

15. Papafragkakis H, Ona MA, Changela $\mathrm{K}$, et al. Acute liver function decompensation in a patient with sickle cell disease managed with exchange transfusion and endoscopic retrograde cholangiography. Therap Adv Gastroenterol 2014;7:217-23.

16. Taher A, Isma'eel H, Mehio G, et al. Prevalence of thromboembolic events among 8,860 patients with thalassaemia major and intermedia in the Mediterranean area and Iran. Thromb Haemost 2006;96:488-91.

17. Sirachainan N Thalassemia and the hypercoagulable state. Thromb Res 2013;132:637-41.

18. Wang M, Zhang M, Li J, et al. Risk factors of portal vein thrombosis in patients with $\beta$ thalassemia major after splenectomy: laparoscopic versus open procedure. Hepatogastroenterology 2014;61:48-54.

19. Guirat-Dhouib N, Mellouli F, Abdelaali A, et al. Budd-Chiari syndrome in a boy with $\beta$ thalassemia major (a study of a new case). J Pediatr Hematol Oncol 2013;35:237.

20. Naik RP, Streiff MB, Haywood C Jr, et al. Venous thromboembolism incidence in the Cooperative Study of Sickle Cell Disease. J Thromb Haemost 2014.

21. Banerjee S, Owen C, Chopra S. Sickle cell hepatopathy. Hepatology 2001;33:1021-8.

22. Gardner K, Suddle A, Kane P, et al. How we treat sickle hepatopathy and liver transplantation in adults. Blood 2014;123:2302-7.

23. Valenti L, Canavesi E, Galmozzi E, et al. Beta-globin mutations are associated with parenchymal siderosis and fibrosis in patients with non-alcoholic fatty liver disease. Journal of hepatology 2010;53:927-33.

24. Esposito BP, Breuer W, Sirankapracha P, et al. Labile plasma iron in iron overload: redox activity and susceptibility to chelation. Blood 2003;102:2670-7.

25. Hershko C. Pathogenesis and management of iron toxicity in thalassemia. Ann N Y Acad Sci 2010;1202:1-9.

26. Cabantchik ZI, Breuer W, Zanninelli G, Cianciulli P. LPI-labile plasma iron in iron overload. Best practice \& research Clinical haematology 2005;18:277-87.

27. Oztas Y, Durukan I, Unal S, Ozgunes N. Plasma protein oxidation is correlated positively with plasma iron levels and negatively with hemolysate zinc levels in sickle-cell anemia patients. Int J Lab Hematol 2012;34:129-35.

28. Mancuso A. Hepatocellular carcinoma in thalassemia: A critical review. World journal of hepatology 2010;2:171-4.
29. Borgna-Pignatti C, Garani MC, Forni GL, et al. Hepatocellular carcinoma in thalassaemia: an update of the Italian Registry. Br J Haematol 2014;167:121-6.

30. Murakami J, Shimizu Y. Hepatic manifestations in hematological disorders. Int J Hepatol 2013;2013:484903.

31. Papastamataki M, Delaporta P, Premetis E, et al. Evaluation of liver fibrosis in patients with thalassemia: the important role of hyaluronic acid. Blood cells, molecules \& diseases 2010;45:215-8.

32. El-Shabrawi MH, Zein El Abedin MY, Omar N, et al. Predictive accuracy of serum hyaluronic acid as a non-invasive marker of fibrosis in a cohort of multi-transfused Egyptian children with $\beta$-thalassaemia major. Arab J Gastroenterol 2012;13:45-8.

33. Poustchi H, Eslami M, Ostovaneh MR, et al. Transient elastography in hepatitis $\mathrm{C}$ virus-infected patients with $\beta$-thalassemia for assessment of fibrosis. Hepatol Res 2013;43:1276-83.

34. Di Marco V, Bronte F, Cabibi D, et al. Noninvasive assessment of liver fibrosis in thalassaemia major patients by transient elastography (TE) - lack of interference by iron deposition. British journal of haematology 2010;148:476-9.

35. Fraquelli M, Cassinerio E, Roghi A, et al. Transient elastography in the assessment of liver fibrosis in adult thalassemia patients. American journal of hematology 2010;85:564-8.

36. Hamidieh AA, Shazad B, Ostovaneh MR, et al. Noninvasive Measurement of Liver Fibrosis Using Transient Elastography in Pediatric Patients with Major Thalassemia Who Are Candidates for Hematopoietic Stem Cell Transplantation. Biol Blood Marrow Transplant 2014.

37. Koh C, Turner T, Zhao X, et al. Liver stiffness increases acutely during sickle cell vaso-occlusive crisis. Am J Hematol 2013;88:E250-4.

38. Deugnier Y, Turlin B, Ropert M, et al. Improvement in liver pathology of patients with $\beta$-thalassemia treated with deferasirox for at least 3 years. Gastroenterology 2011;141:1202-11, 1211.e1-3.

39. Karam LB, Disco D, Jackson SM, et al. Liver biopsy results in patients with sickle cell disease on chronic transfusions: poor correlation with ferritin levels. Pediatr Blood Cancer 2008;50:62-5.

40. Brousse V, Buffet P, Rees D. The spleen and sickle cell disease: the sick(led) spleen. Br J Haematol 2014;166:165-76.

41. Blinder MA, Geng B, Lisker-Melman M, et al. Successful orthotopic liver transplantation in an adult patient with sickle cell disease and review of the literature. Hematol Rep 2013;5:1-4.

42. Mancuso A, Perricone G. Time to define a new strategy for management of hepatocellular carcinoma in thalassaemia? Br J Haematol 2014.

43. Cappellini MD, Motta I, Musallam KM, Taher AT. Redefining thalassemia as a hypercoagulable state. Ann N Y Acad Sci 2010; 1202:231-6. 